Infusionstherapie 1988;15:178

\title{
Impressum, Vol. 15, No. 5, 1988
}

\section{Herausgeber}

S. Karger Verlag für Medizin und Naturwissenschaften GmbH, Postfach 1724, D-8034

Germering

Presserechtlich verantwortlich: Walter Kunz, Gesellschafter

Gründungsherausgeber

H. Reissigl, w. Hofrat

Zentralinstitut für Bluttransfusion und Immunologiscbe Abteilung der Universitätsklinik Innsbruck an der Chirurgischen Universitätsklinik, Anichstr. 35, A-6020 Innsbruck

Schriftleitung

Hauptschriftleiter

K. H. Bäßler, Physiologisch-Chemisches Institut, Johannes-Gutenberg-Universität, Saarstr. 21, D-6500 Mainz Schriftleiter

A. Grünert, Universität Ulm, Zentrum für Anästhesiologie, Oberer Eselsberg-M 23, D-7900 Ulm

K.Widhalm, Universitäts-Kinderklinik, Währinger Gürtel 74-76, A-1097 Wien

Wissenschaftlícher Beî́rat

F. W. Ahnefeld, Ulm (Anästhesie); K. H. Bäßler, Mainz (Physiologische Chemie); H. Bergmann, Linz (Transfusionsmedizin/Anästhesie); C. Bode, Köln (Diätetik); C. Burri, Ulm (Chirurgie); P. Deetjen, Innsbruck (Physiologie); W. Dick, Mainz (Anästhesie); G. Dietze, München (Innere Medizin); R. Dölp, Fulda (Anästhesie); H. von Domarus, Mainz (Kieferchirurgie); W. Druml, Wien (Innere Medi zin); J. Eckart, Augsburg (Anästhesie); P. Ferenci, Wien (Gastroenterologie); H. Förster, Frankfurt (Biochemie); M. Gemperle, Genf (Anästhesie); R. Glaser von Roman, Düsseldorf (Diätetik); A. Grü nert, Ulm (Anästhesie); J. M. Hackl, Innsbruck (Anästhesie); W. Haider, Wien (Pathophysiologie); M. Halmagyi, Mainz (Anästhesie); W. Hartig, Leipzig (Chirurgie); O. Heidenreich, Aachen (Pharmakologie); U. Henneberg, Berlin (Anästhesie); E. Holm, Mannheim (Pathophysiologie); G. Hossli, Zurich (Anästhesie); G. Kleinberger, Steyr (Innere Medizin); K. F. Kopp, München (Nephrologie); V. Kretschmer, Marburg (Transfusionsmedizin); F. Krück, Bonn (Innere Medizin); J. Kult, Bad Mergentheim (Nephrologie); H. G. Lasch, Gießen (Innere Medizin); H. Lochs, Wien (Gastroenterologie); D. Löhlein, Dortmund (Chirurgie); H. Mehnert, München (Innere Medizin); J. M. Müller, Köln (Chirurgie); K. Peter, München (Anäs 
thesie); F. Pohlandt, Ulm (Pädiatrie); U. Rabast, Hattingen (Diäte

tik); H. Reissigl, Innsbruck (Chirurgie/Transfusionsmedizin); P. Riederer, Wien (Neurobiologie); H. Rosegger, Graz (Gynäkologie); E. Rossi, Bern (Pädiatrie); E. Roth, Wien (Stoffwechsel); H. P. Schu ster, Hildesheim (Innere Medizin); M. Semsroth, Wien (Anästhesie/ Intensivmedizin); K. Steinbereithner, Wien (Exp. Anästhesie); H. J. Steinhardt, Mainz (Gastroenterologie); H. Stoeckel, Bonn (Anästhe sie); B. Szczygiel, Warschau (Gastroenterologie); W. Vahlensieck, Bonn (Urologie); R. Wenger, Wien (Innere Medizin); K. Widhalm, Wien (Pädiatrie); F. Willig, Heidelberg (Diätetik); G. Wolfram, München (Ernährungswissenschaft).

Erscheinungsweise

Die Zeitschrift erscheint zweimonatlich; pro Jahr erscheint 1 Band zu je 6 Heften.

Abonnementpreis

Bezugspreis für Jahrgang 15, 1988 DM 148,-/öS 1036,-/SFr. 116,-. 1 Einzelheft kostet DM 28,/öS 196,-/SFr. 22,-, einschließlich MwSt., zuzüglich Postgebühren.

Der Abonnementpreis ist im voraus zahlbar. Das Abonnement der Zeitschrift läuft weiter, wenn es nicht spätestens 4 Wochen vor Abschluß eines Bandes abbestellt wird.

Abonnementbestellungen können bei jeder Buchhandlung, bei der Post oder direkt beim Verlag aufgegeben werden: Bundesrepublik DeutschlandlÖsterreich: S. Karger GmbH, Postfach 1724, D-8034 Germering/München, Tel.: (089) 844021, Telex: 524865 D, Telefax: 8418083, Postscheckkonto: München 40080-807 Schweiz: S. Karger AG, Allschwilerstr. 10, Postfach, CH-4009 Basel, Tel.: (061) 390880, Telex 62652 CH

Manuskripte

Diese sind direkt an den Verlag zu senden; wegen Einzelheiten siehe unter «Hinweise für Autoren» in diesem Heft.

Anzeigen

S. Karger Verlag für Medizin und Naturwissenschaften GmbH, Postfach 1724, D-8034 Germering, Telefon (089) 844021. Gültig ist die Preisliste Nr. 8 vom 1.10.1986.

Für den Inhalt außerhalb des redaktionellen Teiles (insbesondere Anzeigen, Industrieinformationen, Pressezitate, Kongreßinformatio-nen usw.) übernehmen Schriftleitung, Beirat und Verlag keine Ge-währ.

Eine Markenbezeichnung kann warenzeichenrechtlich geschützt sein, auch wenn bei ihrer Verwendung in dieser Zeitschrift das Zeichen ${ }^{\circledR}$ oder ein anderer Hinweis auf etwa bestehende Schutzrechte fehlen sollte. Für Satzfehler, insbesondere bei Dosierungsangaben, wird keine Gewähr übernommen.

Alle Rechte, insbesondere das Recht der Vervielfältigung und Mikro-kopie sowie der Übersetzung, vorbehalten. Nachdruck, auch auszugs-weise, nur mit Genehmigung des Verlages. Fotokopien dürfen nur für den persönlichen Gebrauch als Einzelkopien hersgestellt werden. Jede im Bereich eines gewerblichen Unternehmens zulässig hergestell-te oder benutzte Kopie dient gewerblichen Zwecken gem. § 54(2) UrhG und verpflichtet zur Gebührenzahlung an die Verwertungs-gesellschaft WORT, Abt. VG Wissenschaft, Goethestraße 49, D-8000 München 2. (C) Copyright 1988 by S. Karger Verlag für Medizin und Naturwissenschaften GmbH, Postfach 1724, D-8034 Germering Satz und Druck: Walter Biering GmbH Grafischer Betrieb 
Freisinger Landstr. 21, D-8000 München 45

\section{DAKI}

$\mathrm{O}$

Offizielles Organ

der Deutschen Arbeitsgemeinschaft

für künstliche Ernährung (DAKE)

AKE

,rbeitsgemeinsdiaft für kiinisdie Ernährung

Offizielles Organ

der Österreichischen Arbeitsgemeinschaft

für klinische Ernährung (AKE)

Offizielles Organ der Deutschen Gesellschaft für Transfusionsmedizin und Immunhämatologie 